\title{
Quality-improved and secure multicast delivery method in mobile IPv6 networks
}

\begin{abstract}
With widespread deployment of multicast over Wireless Local Area Networks (WLANs), several issues including fixed data rate transmission, multicast key distribution security, and overlapped multicast address have to be addressed for accommodating an efficient multicast scheme for WLANs. The latter problem can be addressed by utilizing Internet Protocol version 6 (IPv6) which provides significantly more address space compare to existing IPv4. However, in multicast IPv6 over WLANs, when a mobile moves to the border of the multicast group, the data are transmitted at the lowest base rate to support more coverage area, leading to poor Quality of Service (QoS). In this paper, a novel multicast data delivery method over WLANs based on IPv6 protocol is proposed to overcome the problem of fixed base rate and security key distribution in WLANs. Specifically, the proposed method dictates a WLAN Access Point (AP) to encapsulate the multicast packets into unicast Medium Access Control (MAC) packets, and subsequently forward them to the mobile host. In addition, the AP is also responsible for updating and distributing security keys whenever a join or leave operation occurs. The results from our test-bed indicate that the proposed method significantly improve the QoS metrics (i.e., throughput and delay) compared to the existing multicast scenario, as well as able to reduce the amount of generated keys in the networks.
\end{abstract}

Keyword: Data rate transmission; Multicast IPv6; Multicast over WLANs; QoS 